\section{ENT pathology and diplopia}

\author{
Abstract \\ ENT pathology causes diplopia in a minority \\ of patients. Because the various ENT \\ conditions that can produce diplopia are \\ relatively unusual they often escape early \\ detection. We describe the various ENT \\ disorders that can produce diplopia and \\ illustrate this with our experience between \\ 1992 and 1998. We wish to emphasise the \\ benefit which can be obtained from \\ undertaking an ENT history and examination \\ in arriving at the correct diagnosis.
}

Key words Diplopia, Nasal, Sinus, Vision

A. Muneer

N.S. Jones

P.J. Bradley

Department of

Otorhinolaryngology

Head and Neck Surgery

Queen's Medical Centre

Nottingham, UK

\section{R. Downes}

Department of

Ophthalmology

Queen's Medical Centre

Nottingham, UK

Mr N.S. Jones, MD, FRCS Department of

Otorhinolaryngology, Head and Neck Surgery

Queen's Medical Centre University Hospital Nottingham NG7 2UH, UK

Fax: +44 (0)1159709748

e-mail: nick.jones

@nottingham.ac.uk are misaligned; as a presenting symptom it is associated with either local pathology, systemic disorders or a breakdown of local squint.

Spontaneous resolution occurs in the majority of patients presenting with acute non-traumatic diplopia. Whilst the majority of patients with diplopia present directly to ophthalmologists, there are several disorders associated with ENT pathology which the ophthalmologist should be aware of. Early recognition of these disorders is important as diagnostic delay can lead to unnecessary investigations and greater morbidity. A careful history and examination together with an appreciation of these conditions will reduce diagnostic delay and lead to an appropriate and timely referral.

Prospective studies investigating the aetiology of consecutive patients presenting with diplopia have found that the commonest cause is infranuclear cranial nerve palsies secondary to diabetes or microvascular disease. ${ }^{1}$ Other common causes include trauma, ${ }^{2}$ extraocular muscle pathology such as thyroid eye disease and myasthaenia gravis, and supranuclear lesions. A review of the ophthalmological literature reveals few reports relating to the ENT conditions that can produce diplopia; hence these conditions can pass unrecognised until they reach an advanced stage.
A. MUNEER, N.S. JONES, P.J. BRADLEY, R. DOWNES

\section{Method}

We present a review of patients seen in our hospital over a 6 year period (1992-8) in whom diplopia or other ophthalmological symptoms were the presenting symptom secondary to an underlying ENT lesion. Table 1 lists the ENT disorders that enter the differential diagnosis of diplopia. These will be discussed in turn and Table 2 lists the incidence in our experience of benign (1992-8) and malignant intranasal pathology (1983-7).

Table 1. ENT conditions that may present with diploplia

Benign paranasal sinus conditions

Mucocoeles

Osteoma

Fibrous dysplasia

Nasal dermoid

Nasal polyposis

Inverted papilloma

Angiofibroma

Cholesterol granuloma
Malignant paranasal sinus conditions

Primary malignancy

Nasopharyngeal carcinoma

Carcinoma of the paranasal sinuses

Lymphoma

Rhabdomyosarcoma

Malignant melanoma

Extramedullary plasmacytoma

Other rarer malignancies

Metastases

Renal, breast, prostate, lung, plasmacytoma

Trauma and iatrogenic

Blow-out fractures

Damage to the medial canthal ligament, trochlea or orbital muscles

\section{Infection}

Orbit

Retro-orbital

Orbital apex syndrome

Subperiosteal abscess

Intracranial

Lateral sinus thrombosis

Otitic hydrocephalus

Fungal infection

Aspergillosis

Mucormycosis invading the skull base

Granulomatous conditions

Wegener's granulomatosis

Sarcoid

Miscellaneous

Idiopathic collapse of orbital floor 
Table 2. The incidence of ENT pathology determined to be the cause of ophthalmological symptoms in a unit serving a population of 850000 (benign pathology 1992-8 and malignant intranasal pathology 1983-7)

\begin{tabular}{|c|c|c|c|c|c|c|c|}
\hline Diagnosis & No. & $\begin{array}{l}\text { No of eyes } \\
\text { affected }\end{array}$ & Diplopia & Proptosis & Epiphora & $\begin{array}{l}\text { Registered } \\
\text { blind }\end{array}$ & $\begin{array}{c}\text { Ptosis or } \mathrm{CN} \\
\text { palsy }\end{array}$ \\
\hline \multicolumn{8}{|l|}{ Benign } \\
\hline Mucocoeles & 58 & 13 & 7 & 5 & 4 & & 3 \\
\hline Osteoma & 23 & 5 & 5 & 2 & 1 & & \\
\hline Inverted papilloma & 26 & & 2 & & 1 & & \\
\hline Fibrous dysplasia & 3 & 2 & 2 & & & & \\
\hline Paget's disease & 1 & 1 & 1 & & & & \\
\hline \multicolumn{8}{|l|}{ Malignant } \\
\hline Nasopharyngeal Ca & 6 & 4 & 4 & & & & 4 \\
\hline Adenoid cystic $\mathrm{Ca}$ & 17 & 8 & 4 & 7 & 1 & & 5 \\
\hline Squamous cell $\mathrm{Ca}$ & 16 & 9 & 2 & 3 & 2 & 1 & 5 \\
\hline Undifferentiated $\mathrm{Ca}$ & 39 & 13 & 1 & 10 & 8 & & 7 \\
\hline Moderately differentiated $\mathrm{Ca}$ & 6 & 2 & 1 & 1 & 1 & & \\
\hline Adenocarcinoma & 4 & 1 & & & & 1 & 1 \\
\hline Neuroendocrine tumour & 1 & 1 & & 1 & & & 1 \\
\hline Lymphoma & 23 & 11 & 2 & 9 & 4 & 2 & 4 \\
\hline Rhabdomyosarcoma & 6 & 2 & & & & & 2 \\
\hline Malignant melanoma & 22 & 7 & 2 & 4 & 5 & & 1 \\
\hline Extramedullary plasmacytoma & 2 & 1 & 1 & & 1 & & \\
\hline Olfactory neuroblastoma & 5 & 1 & & 1 & & & \\
\hline Other rare malignancies & 13 & 6 & 4 & & 3 & & 1 \\
\hline Metastases & $2+$ & 2 & 2 & 2 & & & \\
\hline \multicolumn{8}{|l|}{ Infection } \\
\hline Subperiosteal abscess & $>16$ & $>16$ & & $>16$ & & 1 & \\
\hline Lateral sinus thrombosis & & 4 & 4 & & & & 4 \\
\hline Otitic hydrocephalus & & 2 & & & & & 2 \\
\hline Petrositis & & 1 & 1 & & & & 1 \\
\hline Paranasal aspergillosis & & 2 & 2 & 2 & & & \\
\hline \multicolumn{8}{|l|}{ Granulomatous conditions } \\
\hline Wegener's granulomatosis & 53 & 25 & 4 & 2 & & & 2 \\
\hline Sarcoid & 16 & 3 & & & 2 & & 1 \\
\hline \multicolumn{8}{|l|}{ Miscellaneous } \\
\hline Idiopathic collapse of orbital floor & 3 & 3 & 3 & & & & \\
\hline
\end{tabular}

$\mathrm{CN}$, cranial nerve; $\mathrm{Ca}$, carcinoma.

\section{Benign paranasal sinus tumours}

Mucocoeles are epithelial-lined lesions containing inspissated mucus. As these lesions expand, adjacent structures are involved. Common symptoms reported include a chronic frontal headache, retro-orbital pain, ocular displacement, ocular palsy and visual failure. ${ }^{3}$ The array of visual symptoms leads to approximately $70 \%$ of patients presenting to the ophthalmology department. ${ }^{3}$

Mucocoeles most frequently originate from the frontal and ethmoidal sinuses and in a review of our series of 58 patients only $7(12 \%)$ had symptoms of diplopia on presentation, ${ }^{4}$ whereas Lund ${ }^{5}$ reported that in her series of 135 frontoethmoidal mucocoeles $95 \%$ had diplopia as one of the presenting symptoms, although the symptoms were often minimal in those who had had the condition for many years. We found that periorbital swelling was a far more common presenting symptom, as was a sensation of pressure or pain, and 10 patients $(19 \%)$ presented acutely with a pyocoele. Sphenoid sinus mucocoeles are encountered relatively infrequently and can involve cranial nerves II to VI with pupil-sparing oculomotor nerve palsy being the most common. This diagnosis is difficult to make at initial presentation; however, patients commonly have a previous history of nasal polyposis or headache, ${ }^{6}$ but there is often little else to direct the clinician to the diagnosis. Where there is a history of nasal polyps, loss of sense of smell, or a II or VI nerve palsy the investigation of choice is $\mathrm{CT}^{7,8}$ (Fig. 1).

Other benign tumours can cause diplopia; not only are they more uncommon but they only occasionally produce diplopia by a mass effect. Osteoma normally grows so slowly that the patient does not complain of diplopia, even with marked global displacement (Fig. 2). Nasal dermoids medial to the medial canthus normally present in children with a cosmetic deformity before they produce diplopia. Nasal polyposis, particularly in association with aspirin sensitivity, can be so extensive that the nasal bones are expanded and erode through the attachment of the medial canthal ligament. Inverted papilloma can expand the paranasal sinuses and displace the globe. The majority are locally invasive nasal polyps although $8-15 \%$ are associated with malignancy.

Examination of the nose will normally reveal unilateral nasal polyps. Angiofibroma, which normally presents in 8- to 15-year-old boys and usually originates in the pterygopalatine fossa, can displace the orbit and invade the skull base producing lesions involving cranial nerves III-VI. There is unilateral nasal obstruction and a blue or 


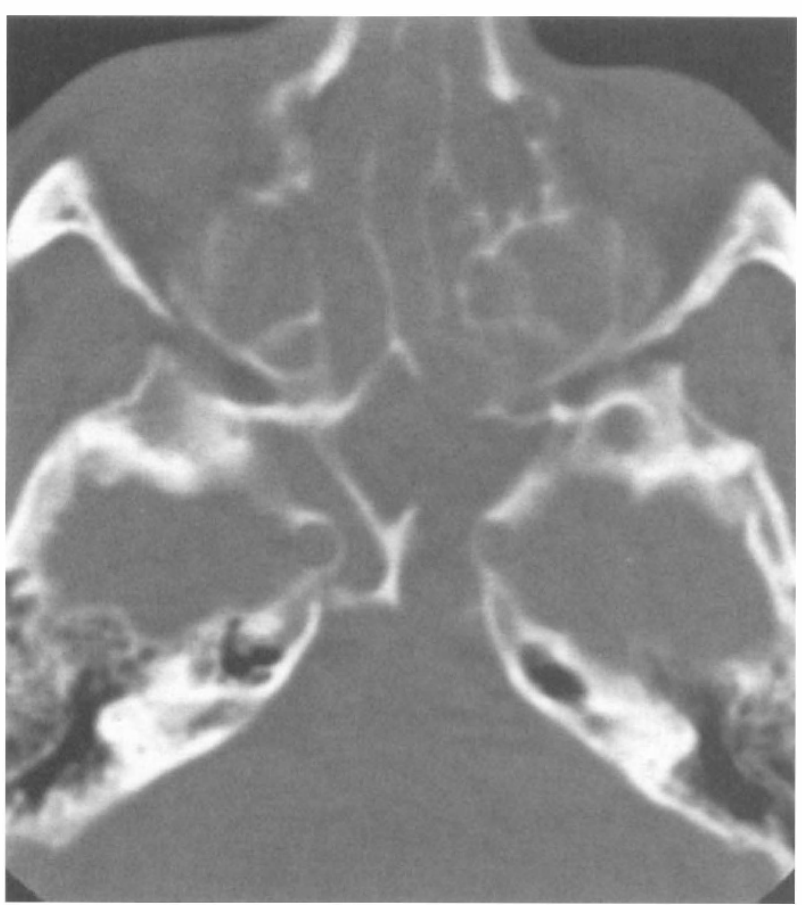

Fig. 1. Axial CT scan showing a sphenoidal mucocoele in a patient with polyposis. The posterior wall of the sphenoid is eroded and the patient presented with a VI nerve palsy.

magenta-coloured mass in the nasal airway. Cholesterol granuloma can expand any sinus and displace the globe and is atypical when compared with most other paranasal sinus conditions in that apart from producing expansion of a sinus, it is silent or asymptomatic. Apart from asking about nasal symptoms and examining the nose, a CT scan is the investigation of choice.

\section{Malignant paranasal sinus conditions}

Nasopharyngeal carcinoma is prevalent in Far Eastern countries (particularly the southern provinces of China). It is infrequently encountered in the Caucasian population and then in those over the age of 50 years with a neck mass, headache, epistaxis, tinnitus, hearing impairment and nasal obstruction. Cranial nerve

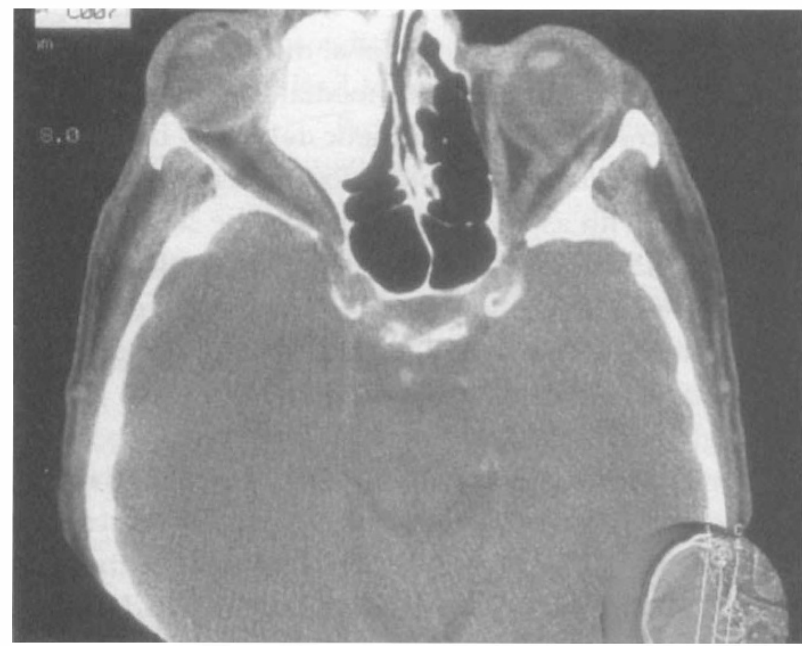

Fig. 2. Axial CT scan showing a large osteoma of the ethmoid sinuses.

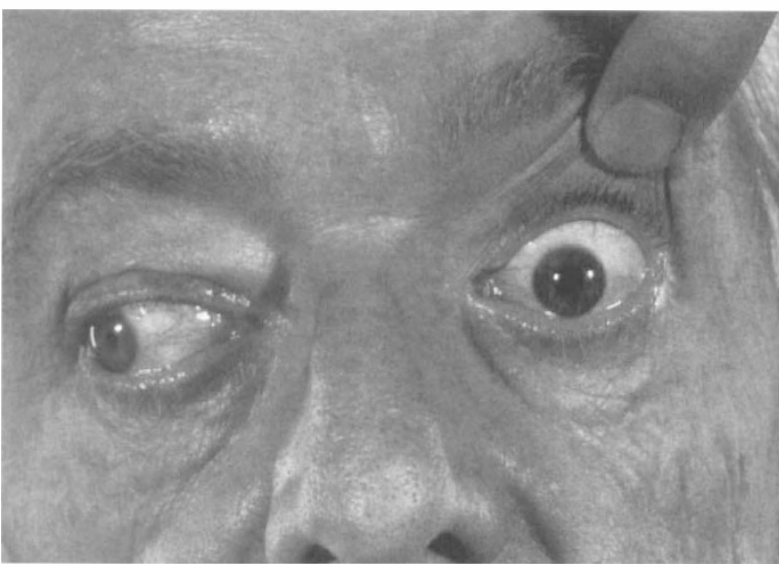

Fig. 3. A left III nerve palsy due to a nasopharyngeal carcinoma.

involvement with associated diplopia signifies a poor prognosis and occurs in approximately $20-25 \%$ of all nasopharyngeal carcinoma cases. ${ }^{9}$ The cranial nerves most commonly involved are abducens, trigeminal and oculomotor nerves (Figs. 3, 4). Cranial nerve lesions can occur in the absence of neck lymphadenopathy or any obvious nasopharyngeal mass, thus further delaying the diagnosis. Radiotherapy can provide temporary control of the disease in over half of patients, with an occasional dramatic remission in a minority.

Carcinoma of the paranasal sinuses usually presents late, with $75 \%$ of patients with squamous cell carcinoma of the maxilla being $\mathrm{T} 3$ or T4 (AJC/UICC classification) when they are first seen. The presenting symptoms may be due to its expansion out of its bony surround, into the orbit (Fig. 5) with diplopia or exophthalmos, the mouth

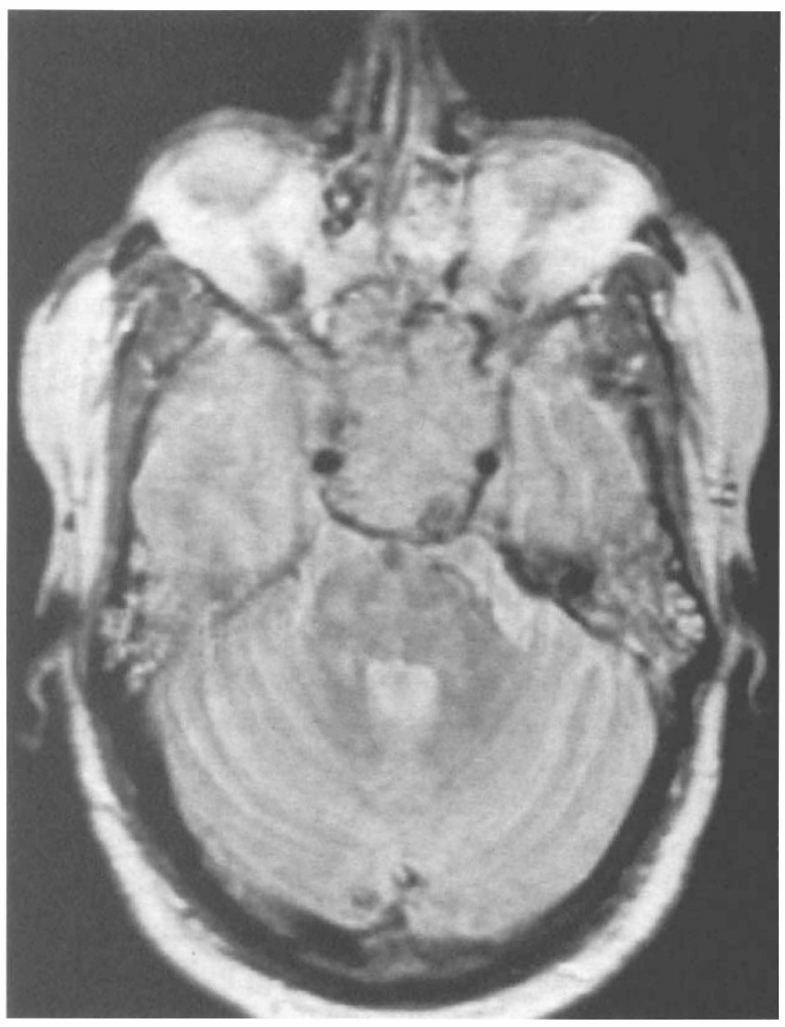

Fig. 4. Axial MRI scan showing a nasopharyngeal carcinoma invading the skull base. 


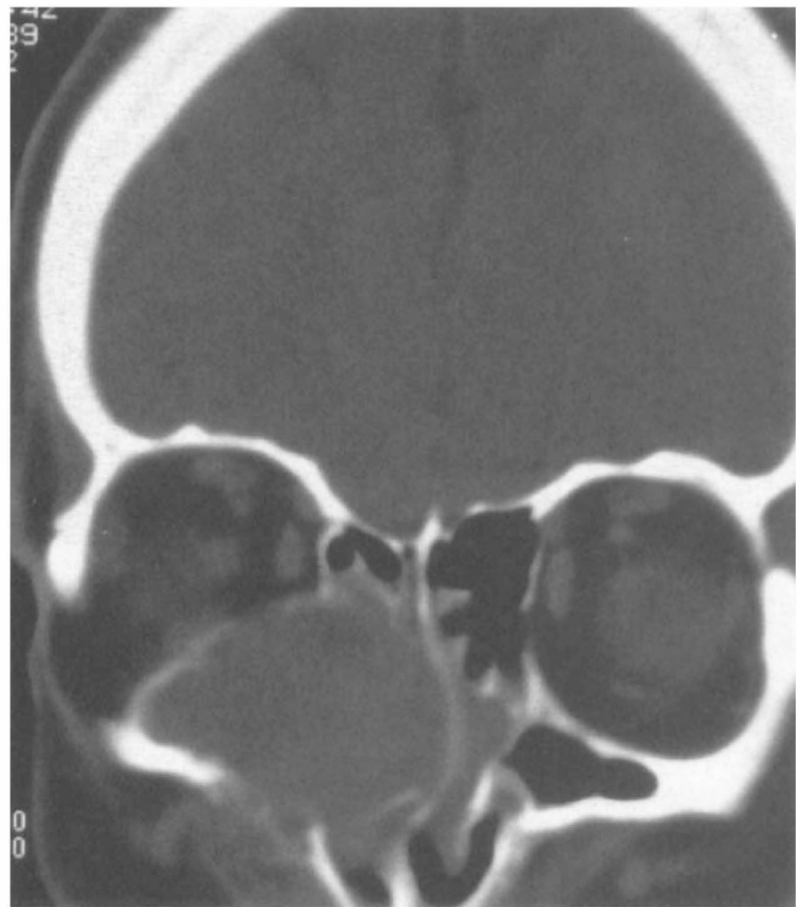

Fig. 5. Coronal CT scan of a low-grade adenocarcinoma of the maxillary sinus that presented with epiphora and diplopia.

with loose teeth, the cheek with infraorbital paraesthesia or swelling, or the pterygopalatine fossa with trismus and cranial nerve signs. It may also produce epiphora. Unilateral nasal obstruction or a purulent bloody discharge should alert the clinician to the diagnosis.

A range of primary malignancies occur in the paranasal sinuses, including squamous cell carcinoma, adenocarcinoma (which is more prevalent in workers of hard wood), olfactory neuroblastoma (Fig. 6) and adenoid cystic carcinoma amongst others.

Primary malignant melanoma of the nasal and paranasal cavities usually originates from the nasal septum and lateral nasal wall followed by the middle and inferior turbinates. ${ }^{10}$ Approximately $20 \%$ of all melanomas occur in the head and neck region, with $6.3 \%$

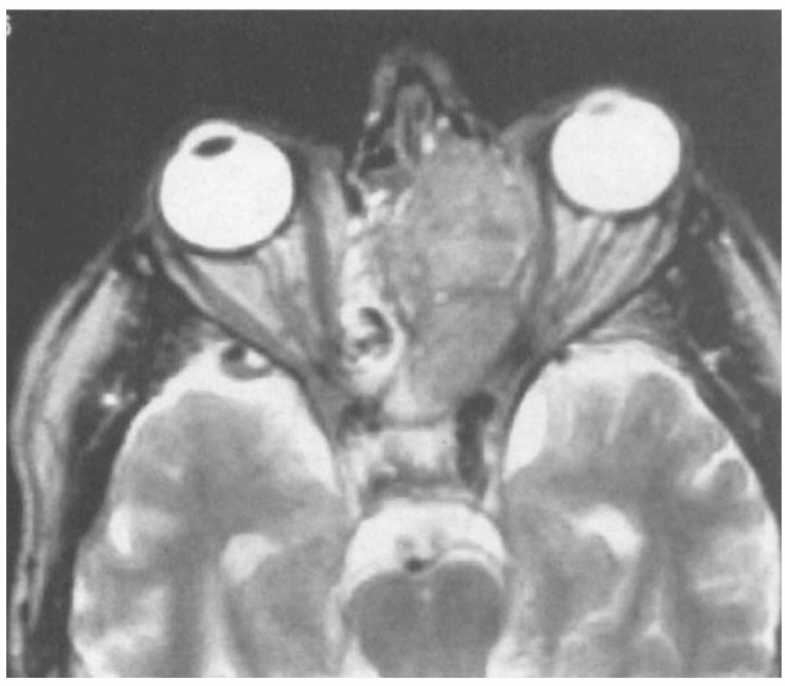

Fig. 6. Axial MRI scan showing an olfactory neuroblastoma that presented with diplopia and unilateral nasal obstruction.

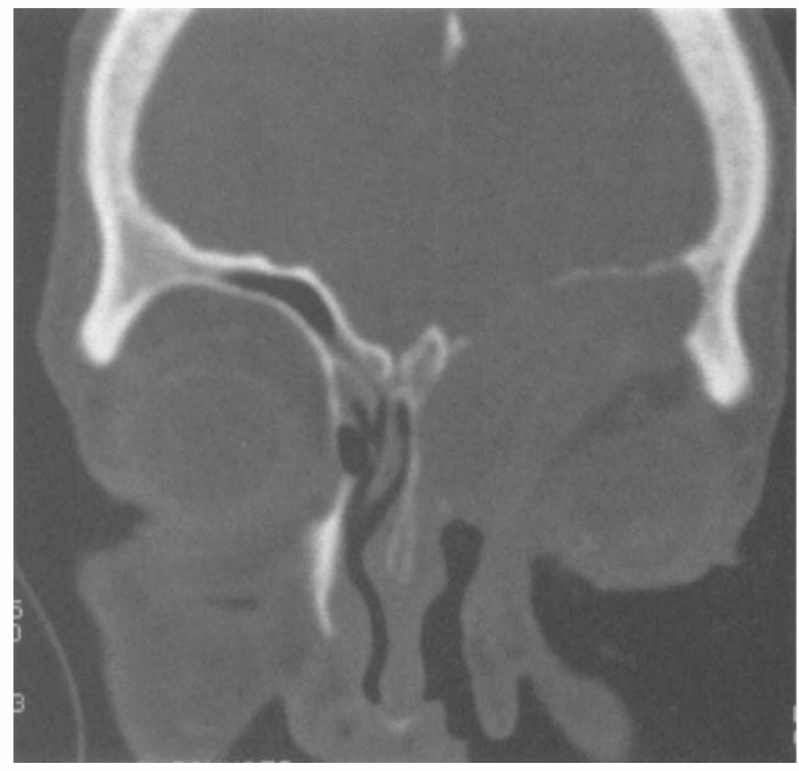

Fig. 7. Coronal CT scan showing a non-pigmented malignant melanoma of the paranasal sinuses producing diplopia.

occurring in the mucous membranes. ${ }^{11}$ The presenting symptoms are initially innocuous and often localised to the nasal cavity to produce nasal obstruction, nasal discharge, epistaxis and swelling, although headaches are also reported. Ocular symptoms are not commonly reported but do occur (Fig. 7). In a previous review of 39 cases, epiphora was a more frequent complaint than diplopia. $^{12}$

\section{Metastases}

Neoplastic disease of the orbit is most commonly due to metastatic tumour. ${ }^{13}$ A review of 213 patients with known metastatic disease found that primary neoplasms in the lung and breast were most likely to be associated with metastatic disease to the orbit. ${ }^{14}$ The onset of diplopia commonly leads to previously undiagnosed patients presenting to the ophthalmology department. Features of the underlying primary tumour may be apparent on initial presentation but this is not always the case (Fig. 8). The most common primary tumours that

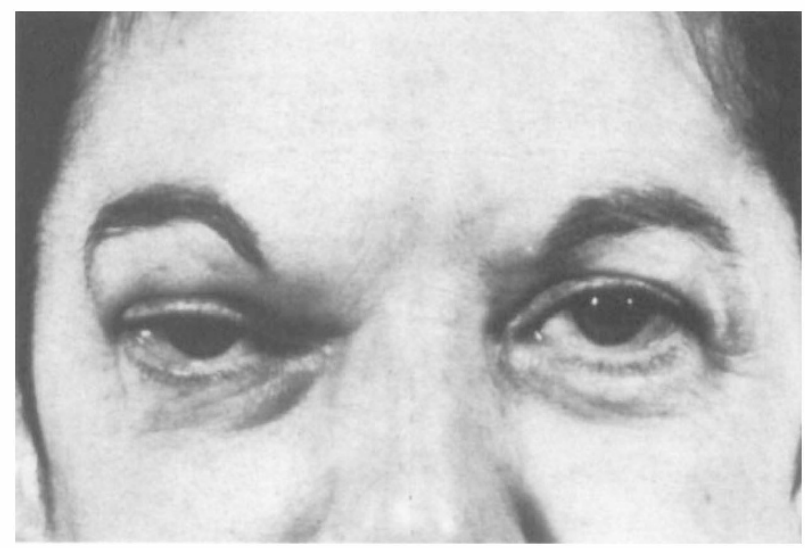

Fig. 8. A solitary renal metastatis involving the right frontal sinus and displacing the orbit producing diplopia. 


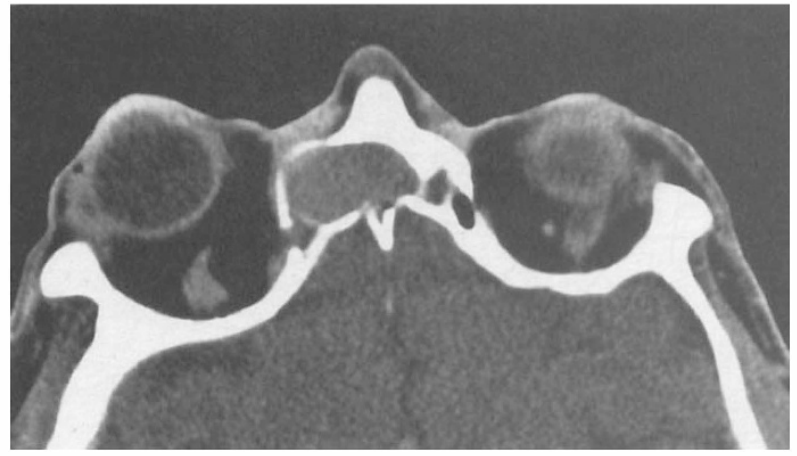

Fig. 9. Axial CT scan showing a plasmacytoma that presented with diplopia in a patient with known multiple myeloma.

metastasise to the paranasal sinuses are breast, lung, prostate renal and medullary or extramedullary plasmacytoma.

A plasmacytoma produces a painless unilateral proptosis associated with impaired vision and diplopia (Fig. 9) occasionally with bilateral orbital involvement. ${ }^{15}$ Extramedullary plasmacytomas of the head and neck can occur and involve the nasal cavity and paranasal sinuses, accounting for $80-90 \%$ of plasmacytomas occurring at extramedullary sites. Up to $60 \%$ of patients with an osseous lesion will eventually develop multiple myeloma.

\section{Granulomatous conditions}

Wegener's granulomatosis is a multisystem necrotising vasculitis that predominantly affects the upper and lower respiratory tracts and kidneys. Previous reports have stated that up to $95 \%$ of sufferers have inflammatory changes in the paranasal sinuses and upper airways with long-term sequelae including septal perforation and saddle nose deformities. ${ }^{16}$ Ocular manifestations of Wegener's granulomatosis can occur early in the form of scleritis, episcleritis, corneal ulceration and uveitis. In addition to this unilateral and bilateral proptosis, chemosis and reduced extraocular movements as well as cranial nerve involvement can lead to diplopia (Fig. 10) or an intraorbital mass (Figs. 11,12). It is worth taking an ENT history and examining the nose in these patients as it will often help in making a diagnosis (Fig. 13).

In our review of 53 consecutive patients with Wegener's granulomatosis we found 25 had ophthalmic symptoms and of these 2 presented with diplopia. The

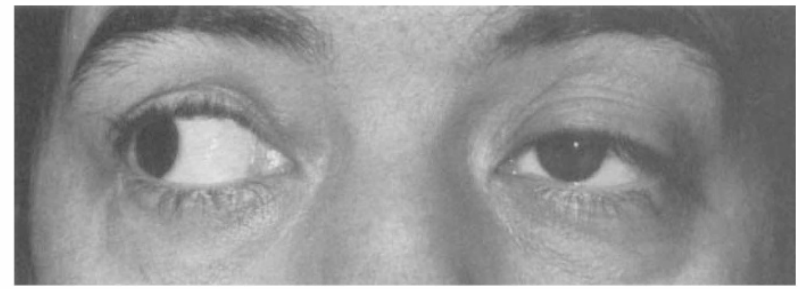

Fig. 10. A left ophthalmoplegia due to Wegener's granulomatosis involving III-VI cranial nerves around the cavernous sinus. A diagnosis was made after inspection and biopsy of nasal mucosa.

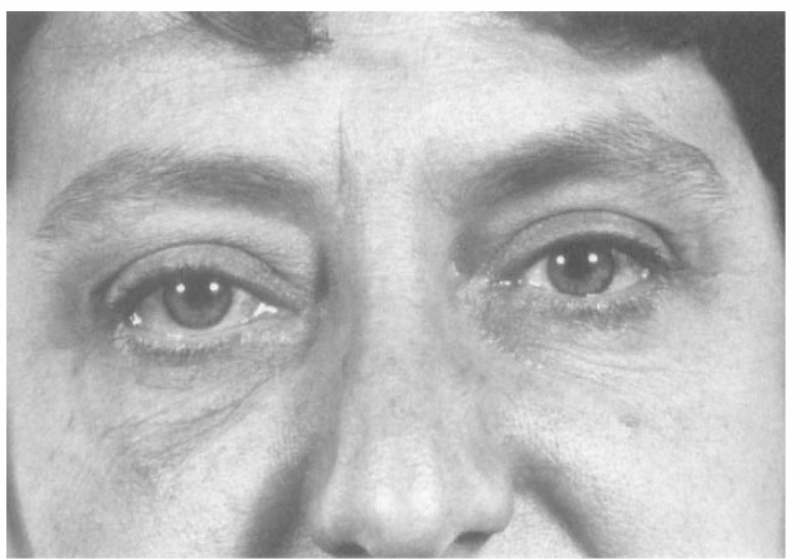

Fig. 11. Right-sided proptosis due to an orbital mass from Wegener's granulomatosis.

diagnosis can be made by serological assay of cytoplasmic anti-neutrophil cytoplasmic antibody, although in 5 of our patients this was initially negative, becoming positive 3 months to 5 years later. Biopsy of nasal mucosa may be helpful if the tissue is macroscopically abnormal.

Sarcoidosis can produce nerve palsies, one of the most common being a VI nerve palsy. Intranasally $6 \%$ of patients with sarcoidosis are found to have granulomatous mucosa which often resembles small cobblestones rather than smooth mucosa. There is frequently crusting as in Fig. 13.

\section{Bacterial infection}

A subperiosteal abscess can displace the orbit and theoretically produce diplopia, but patients rarely complain of this as the surrounding oedema normally results in supraorbital swelling with closure of the lids. Pain and toxaemia as well as an inability to open the lids other than to test vision eclipse the presence of diplopia. Occasionally a sterile abscess can form if there is inadequate drainage and antibiotics sterilise the pus (Fig. 14).

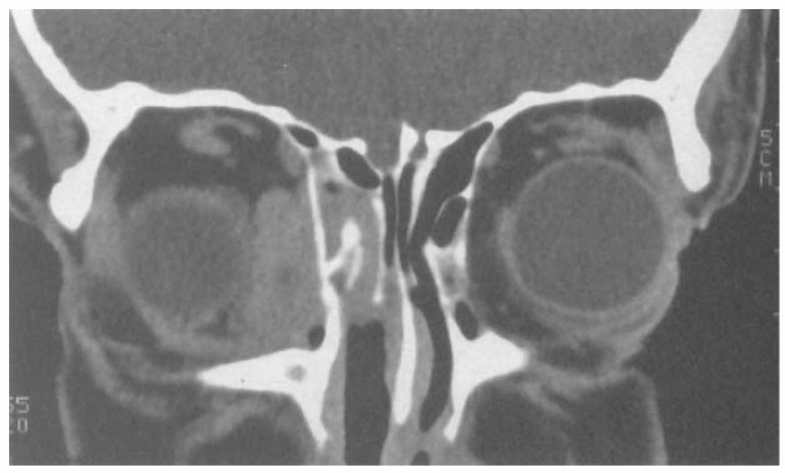

Fig. 12. Coronal CT scan of the patient in Fig. 11. 


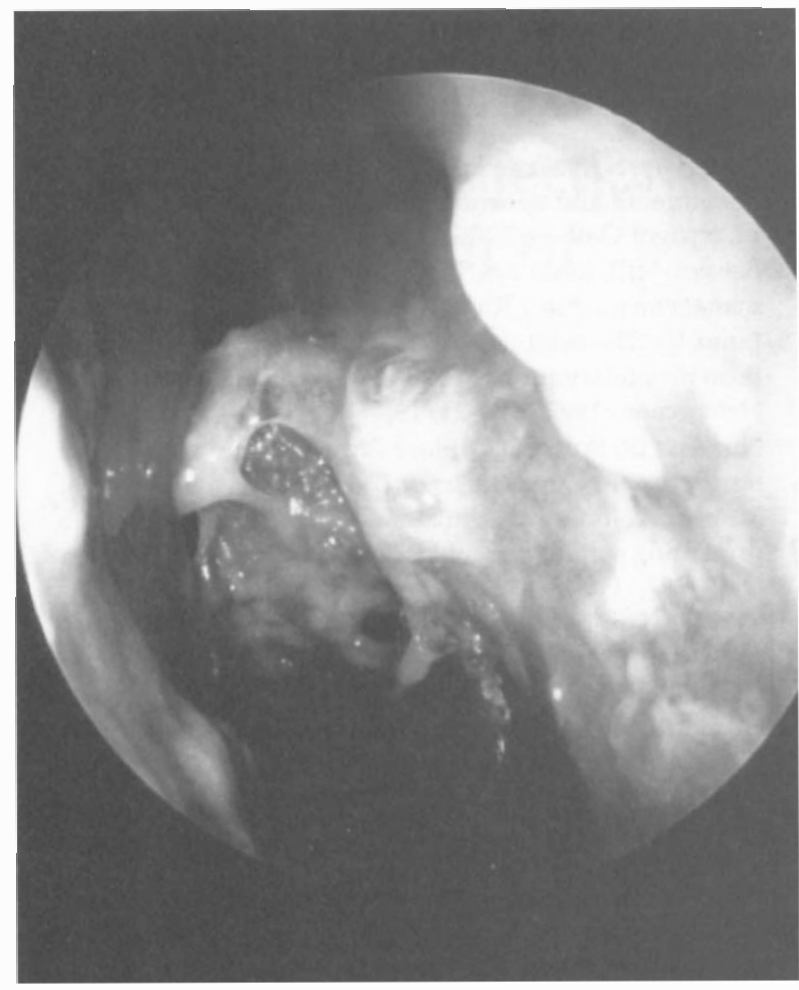

Fig. 13. The classical crusting and granular mucosa of the nasal airway in granulomatous disease.

\section{Petrositis and lateral sinus thrombosis}

Acute mastoiditis and its related complications are now rare with the advent of antibiotics and possibly an improvement in social conditions and nutrition.

However, intracranial complications do still occur. Direct

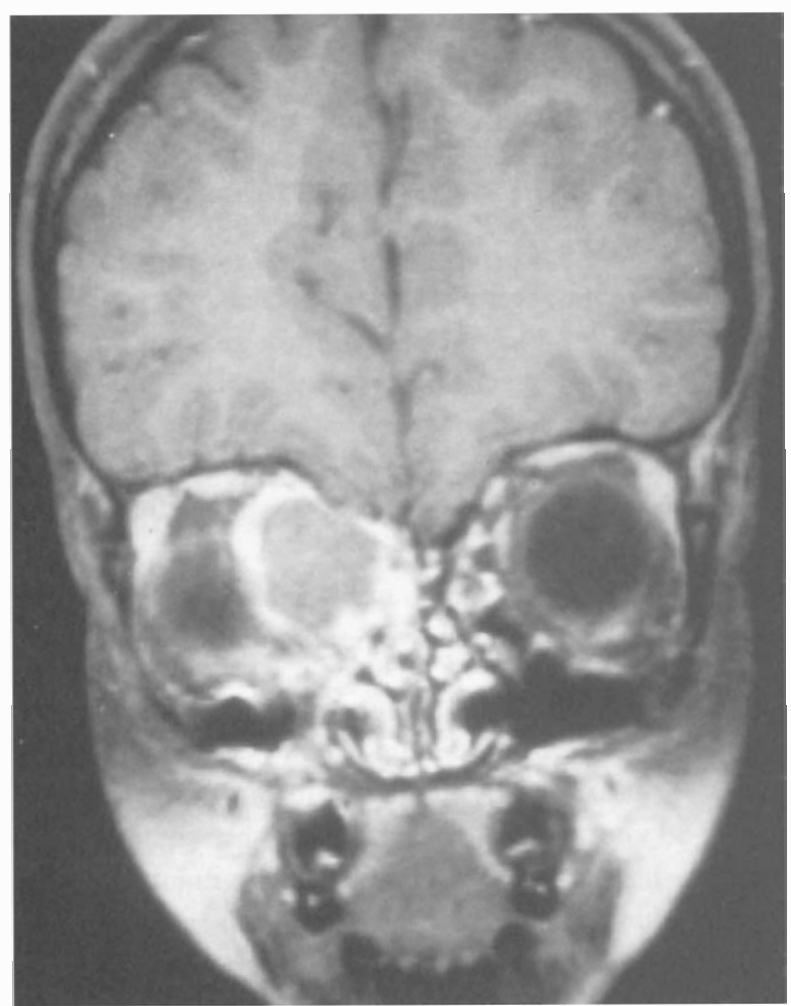

Fig. 14. Coronal MRI scan showing a sterile abscess due to inadequate drainage of a subperiosteal collection of pus. extension of infection from the middle ear or mastoid to the pneumatised petrous bone typically produces a unilateral headache which is usually retro-orbital, supraorbital or temporal. The triad of middle ear infection, retro-orbital pain and abducens nerve palsy with associated diplopia is characteristic of Gradenigo's syndrome. The localised pachymeninigitis due to the petrositis causes inflammation and malfunction of the adjacent abducens nerve. Cranial nerve palsies can also occur in the absence of petrositis. A lateral sinus thrombosis can cause a spreading phlebitis via the inferior petrosal sinus. ${ }^{17}$ Lateral sinus thrombosis can occur per se or be associated with meningitis, brain abscess, extradural empyema or cavernous sinus thrombosis. Patients are systemically unwell as well as having features of diplopia and otalgia on the ipsilateral side.

Otitic hydrocephalus most commonly presents in young children and adolescents following a middle ear infection. Otitic hydrocephalus probably occurs secondary to lateral sinus thrombosis which has spread to the superior sagittal sinus and affects the arachnoid villi, resulting in impaired absorption of cerebrospinal fluid and a raised intracranial pressure. Headache, vomiting, intermittent dizziness, otorrhoea and papilloedema are the most frequently encountered symptoms. Diplopia due to either an ipsilateral or a contralateral abducens nerve palsy can occur; hence this is commonly referred to as a 'false localising sign'. MRI will detect these changes approximately $24 \mathrm{~h}$ before CT.

\section{Aspergillosis}

Aspergillus infection of the nose and paranasal sinuses is associated with immunocompromised patients and diabetics but is also seen in normal healthy individuals. The infection of the paranasal sinuses can be either

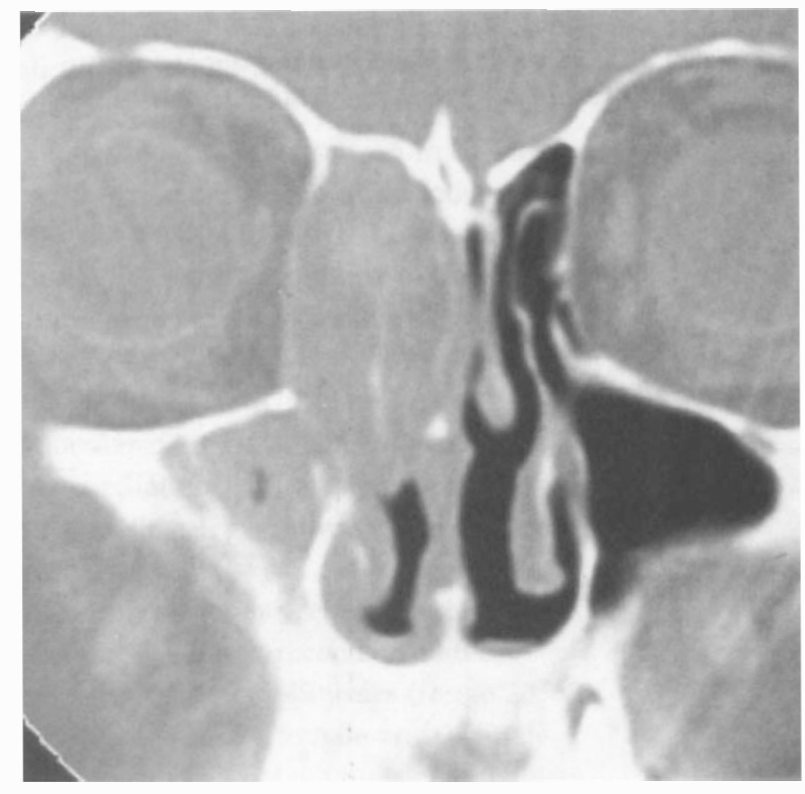

Fig. 15. Coronal $C T$ scan in a patient with diplopia secondary to aspergillosis of the ethmoid sinuses. 
invasive or non-invasive with or without an allergic reaction. Invasive aspergillosis of the paranasal sinuses may present with purulent rhinorrhoea accompanied by nasal obstruction and localised facial discomfort.

However, contiguous spread through the adjacent bone can lead to orbital and intracerebral involvement. It can present with proptosis and epiphora together with reduced visual acuity. A non-invasive aspergilloma can produce displacement of the globe due to expansion with or without bone erosion ${ }^{18}$ (Fig. 15). Unilateral polyps and pus are almost always visible in the nasal airway. Titres of Aspergillus precipitans are raised. A CT scan will show the bony anatomy whereas T1- and T2-weighted MRI scans show the extent of soft tissue and vascular invasion.

\section{Sinusitis feigning orbital disease}

One in three individuals without nasal symptoms will have mucosal thickening found incidentally on their CT scans. ${ }^{19}$ If an individual has no nasal symptoms or signs it is unlikely that they have any significant nasal disease and it is unwise to 'diagnose' sinusitis on the basis of CT findings alone.

\section{Conclusion}

Disease of the paranasal sinuses is only infrequently the cause of diplopia. However, if patients with diplopia have nasal symptoms or signs, the possibility of nasal pathology should be pursued in order not to overlook systemic conditions that can involve both the orbit, globe and nose. Awareness of the nasal conditions that can produce diplopia may alert the clinician to ask about nasal symptoms, examine the nose and therefore help in making a timely and accurate diagnosis.

\section{References}

1. Morris RJ. Double vision as a presenting symptom in an Ophthalmic Casualty Department. Eye 1991;5:124-9.
2. Bullock P, Hewitt S, Jones N, McGurk M, McConachie N, Pitts J, et al. Orbit and eye injuries. In: Jones NS, editor. Craniofacial trauma. Oxford: Oxford University Press, 1997:126-36

3. Moriyama H, Nakajima T, Honda Y. Studies on mucoceles of the ethmoid and sphenoid sinuses: analysis of 47 cases. J Laryngol Otol 1992;106:23-7.

4. Beasley NJP, Jones NS. Paranasal sinus mucoceles: modern management. Am J Rhino 1995;9:251-6.

5. Lund VJ. The orbit. In: Mackay I, Bull TR, editors. ScottBrown's otolaryngology. Vol 4. 6th ed. Oxford: Butterworth Heinemann, 1997:24/1-24/19.

6. Nugent GR, Sprinkle P, Bloor BM. Sphenoid sinus mucoceles. J Neurosurg 1970;72:443-51.

7. Muneer A, Jones NS. Unilateral abducens nerve palsy: a presenting sign of sphenoid sinus mucocoèles. J Laryngol Otol 1997;111:644-6.

8. Setti DS, Lau DP, Chan C. Sphenoid sinus mucocoeles presenting with isolated oculomotor nerve palsy. J Laryngol Otol 1997;111:471-3.

9. Ho JHC. An epidemiological and clinical study of nasopharyngeal carcinoma. Int J Radiat Oncol Biol Phys 1978;4:182-97.

10. Lund V. Malignant melanoma of the nasal cavity and paranasal sinuses. J Laryngol Otol 1982;96:347-55.

11. Conley J, Pack GT. Melanoma of the mucous membranes of the head and neck. Arch Otolaryngol 1974;99:315-9.

12. Freeman MH, Desanto LH, Devine KD, Weiland LH. Malignant melanoma of the nasal cavity and paranasal sinuses. Arch Otolaryngol 1973;97:322-5.

13. Trimble R. Diplopia as a presenting sign of neoplasia. Trans Ophthalmol Soc UK 1980;100:498-500.

14. Albert DM, Rubenstein RA, Scheie HG. Tumour metastasis to the eye: incidence in 213 adult patients with generalised malignancy. Am J Ophthalmol 1967;63:723-6.

15. Hayes JG, Petersen M, Kakulas BA. Multiple myeloma with bilateral orbital infiltration and polyneuropathy. Med J Aust 1980;2:276-7.

16. Sundaresan N, Noronha A, Hirschauer J, Signeira EB. Oculomotor palsy as initial manifestation of myeloma. JAMA 1977;238:2052-3.

17. Homer JJ, Johnson IL, Jones NS. Middle ear infection and sixth nerve palsy. J Laryngol Otol 1996;110:872-4.

18. Daghistani KJ, Jamal TS, Zaher S, Nassif OI. Allergic Aspergillus sinusitis with proptosis. J Laryngol Otol 1992;106:799-803.

19. Jones NS, Strobl A, Holland I. CT findings in 100 patients with rhinosinusitis and 100 controls. Clin Otolaryngol 1997;22:47-51 